\title{
Ansiedade e Depressão de Mães de Recém-nascidos com Malformações Visíveis ${ }^{1}$
}

\author{
Gimol Benzaquen Perosa ${ }^{2}$ \\ Flávia Cristina Pereira Silveira \\ Isabela Cristina Canavez \\ Universidade Estadual Paulista Júlio de Mesquita Filho
}

\begin{abstract}
RESUMO - Este estudo teve como objetivo comparar as condições emocionais de mães cujos filhos nascem com malformações visíveis, em dois momentos: após o nascimento e três meses após alta hospitalar. Para tanto, foram avaliados os sinais de ansiedade e depressão de 17 mães desses recém-nascidos pelo Inventário de Depressão de Beck e Inventário de Ansiedade Traço-Estado - IDATE. Confirmando a literatura, com outras crianças de risco, observaram-se altos índices de ansiedade e depressão no pós-parto imediato, uma redução significativa da ansiedade-estado, da depressão e dos sinais clínicos de ansiedade, três meses após a alta. Pode-se supor que estava ocorrendo uma adaptação gradual dessas mães, que contavam com suporte social e grupo de apoio, à medida que tinham um contato íntimo com a criança e percebiam suas habilidades como cuidadoras. Discutem-se as limitações do estudo e necessidade de investigações orientadas para aprofundar a identificação dos fatores que potencializam ou dificultam a adaptação materna.
\end{abstract}

Palavras-chave: ansiedade materna; depressão materna; bebês malformados.

\section{Anxiety and Depression in Mothers of Newborns with Visible Deformities}

\begin{abstract}
This study aimed at comparing anxiety and depression symptoms in mothers of malformed babies soon after their births and three months after hospital discharge. Seventeen mothers were assessed using Beck Depression Inventory and IDATE State-Trait Anxiety Inventory. These results indicated, as in others studies, that the mothers presented an increased anxiety and depression state soon after delivery and a significant reduction in these conditions and in clinical signs of anxiety three months after discharge. These results support the suggestion that there is a gradual adaptation of the mothers as they have a close contact with the child and a sense of maternal competence. Methodological limitations of the study are discussed as well as and the need of future researches to investigate which factors potentializes or moderated maternal adaptation.
\end{abstract}

Key words: maternal anxiety; maternal depression; malformed babies.

Os avanços nos cuidados médico-hospitalares na área da Neonatologia trouxeram, nos últimos anos, um aumento significativo no índice de sobrevivência de bebês de alto risco, prematuros, baixo peso, doentes ou com malformações. Entretanto, essa sobrevida trouxe uma nova realidade para um grande número de famílias. O nascimento de uma criança de risco produz, na mãe, um impacto na sua representação de um bebê sadio e normal, promove um campo de luta e cria a necessidade de um processo de reconstrução da imagem que ela antecipou durante a gravidez (Pedremônico, 1998). Para Drotar, Baskieuvicz, Irvin, Kennel e Klaus (1975) e Brunhara e Petean (1999), na maioria dos casos há uma aceitação progressiva da criança, mas, até conseguir se reorganizar, os pais passam por uma seqüência de estágios emocionais: choque, tristeza, negação, revolta, resignação e adaptação.

As reações iniciais dos pais, bem como o grau de apego ao filho, dependem, em parte, do tipo de malformação: se ela é visível, se pode ser corrigida, se afeta o sistema nervoso central ou a genitália, se é familiar, visto que essas características definem a longo-termo os problemas que eles terão que enfrentar (Kennel \& Klaus, 1993; Sloper \& Turner, 1993). Entretanto, ainda não há resultados conclusivos que

1 Apoio financeiro da Fapesp.

2 Endereço: Distrito de Rubião Júnior, S/N, Botucatu, SP, Brasil 18618970, Caixa Postal: 540.E-mail: gimol@fmb.unesp.br demonstrem uma relação direta entre a gravidade ou visibilidade das malformações e a dificuldade de reorganização da família. Se nos estudos de Sloper e Turner (1993) e de Kazac e Clark (1986) observou-se que, a preocupação e o embaraço são mais imediatos quanto mais visíveis são os defeitos, Fagan e Schor (1993) não conseguiram replicar os resultados. Para Sloper e Turner (1993), no primeiro ano, a adaptação de pais cujos filhos têm problemas motores é mais difícil do que a de pais cujas crianças têm problemas mentais, pela importância que as respostas motoras têm na qualidade de vida, nesse estágio de desenvolvimento. Para Julian-Renier e cols. (1995), pesquisando os pais de crianças com síndrome de Down, outras variáveis podem dificultar a adaptação, como as crises econômicas, o restrito grupo de apoio devido ao tamanho das famílias e ao crescente número de divórcios, e o pequeno número de serviços públicos para $\mathrm{o}$ atendimento de crianças com problemas.

A vulnerabilidade biológica desses recém-nascidos agrava a dificuldade de adaptação dos pais, pois, logo após o nascimento, a maioria delas é encaminhada para uma unidade de tratamento intensivo (UTI), onde, muitas vezes, o contato com a mãe é restrito e a separação potencializa a percepção de ineficiência em oferecer cuidados adequados ao filho. Quando as crianças saem do hospital, as profundas exigências e cuidados que demandam, comprometem o tempo 


\section{G. B. Perosa e cols}

e energia materna que, associados às incertezas quanto ao futuro, podem ter como conseqüência dificuldades emocionais e a instalação de quadros ansiosos e depressivos (Kennel \& Klaus, 1993; Quentel, 1997).

A depressão materna, que, segundo a literatura, acomete de $10 \%$ a $20 \%$ das puérperas (Moraes \& cols., 2006), tem sua etiologia multideterminada, sofrendo influências genéticas, de mudanças fisiológicas, de estressores psicológicos e do contexto cultural. Caracteriza-se por alterações no apetite, dificuldade de dormir, especialmente depois de amamentar o bebê, crises de choro, desatenção, falta de concentração, falta de energia e dificuldade de engajar-se em atividades que antes eram consideradas agradáveis e a sensação de não ser capaz de lidar com situações novas (Frizzo \& Piccinini, 2005).

Alguns fatores podem agravar o quadro depressivo como a grande frustração das expectativas relacionadas com a maternidade, conflitos conjugais (Maldonado, 2000), baixas condições sócio-econômicas (Coutinho, Martins \& Morais, 2002; Cruz, Simões \& Faisal-Cury, 2005; Moraes \& cols, 2006) e a falta de suporte social (Cruz \& cols., 2005; Fagan \& Schor, 1993; Schwengber \& Piccinini, 2005). Variáveis do próprio bebê também foram identificadas como fator de risco para depressão puerperal. Padovani, Linhares, Carvalho, Duarte e Martinez (2004) associaram o aparecimento de sintomas clínicos de depressão com a prematuridade da criança; Murray, Stanley, Hooper, King e Fiori-Cowley (1996) observaram que a irritabilidade do recém-nascido, assim como seu pobre desempenho motor, são preditores de depressão materna.

Nos dias de hoje, a depressão materna constitui um fator de preocupação nos serviços de saúde pediátrica, pois pode provocar desestruturação familiar e possíveis prejuízos cognitivos e emocionais às crianças sob os cuidados de mães com depressão. Mães deprimidas muitas vezes não ficam emocionalmente disponíveis para o recém-nascido, tendem a ser menos responsivas, o que pode ocasionar privação psicossocial e condições de desenvolvimento adversas (Frizzo \& Piccinini, 2005). Em estudo realizado na Índia, Patel, De Souza e Rodrigues (2003) observaram que a depressão materna estava associada a um atraso do desenvolvimento cognitivo, seis meses após o nascimento. A depressão materna já foi associada, também, com risco de abuso à criança, bem como de trauma não intencional à mesma (Neeldman \& cols., 2000).

Em contraste com a depressão, a ansiedade puerperal (AP), apesar de sua prevalência e importância potencial, tem sido pouco estudada. Os dados sobre a prevalência de ansiedade puerperal variam muito de estudo para estudo, mesmo utilizando os mesmos instrumentos de avaliação. Assim, Britton (2005), utilizando o STAIT (Spielberger State-Trait Anxiety Inventory), obteve uma prevalência de $24,9 \%$ de ansiedade puerperal em uma amostra de mães americanas, antes da alta hospitalar, cujos filhos saudáveis haviam nascido a termo. Utilizando o mesmo instrumento, Faisal-Cury e Menezes (2006) observaram uma prevalência de AP de 44,8 para ansiedade-estado e 46,1 para ansiedade-traço em uma amostra de mães brasileiras atendidas em clínica privada. Maior renda e maior faixa etária da mulher diminuíam o risco de AP, ao passo que presença de intercorrências com o recém-nascido e maior número de filhos aumentavam o risco de AP. Com relação a recém-nascidos de risco, Zanardo e Freato (2001) observaram uma prevalência de ansiedade materna de 47,1 para ansiedade-estado, em mães de crianças nascidas com problemas pulmonares, e Padovani e cols. (2004), utilizando outro instrumento, o Idate, verificaram que $44 \%$ das mães de prematuros apresentavam sintomas clínicos de ansiedade.

A ansiedade é um estado emocional que tem componentes fisiológicos e psicológicos, que abrangem sensações de medo, insegurança e antecipação apreensiva, pensamento dominado por idéias de catástrofe ou incompetência pessoal, aumento do estado de vigília, tensão e dor muscular, sensação de constrição respiratória, tremor e inquietação e vários desconfortos somáticos consequientes da hiperatividade do sistema nervoso autônomo (Gorenstein \& Andrade, 2000). Existem evidências de que tem efeito negativo na galactopoese e sobre a qualidade de vínculo mãe/bebê (Chatterton \& cols., 2000; Faisal-Cury \& Menezes, 2006). Há evidências, inclusive, de repercussões emocionais dos quadros de ansiedade materna, a longo prazo, tanto na mulher, como na criança. Barnett, Scaasfma, Guzman e Parker (1991) identificaram que, cinco anos após o nascimento, mães que apresentaram alta ansiedade no pós-parto tiveram mais patologias psicossociais e suas crianças mais sinais de desadaptação, quando comparadas com mães com baixa ansiedade. Apesar da ansiedade ser um estado emocional que faz parte do espectro normal das experiências humanas, funcionando como um alerta que possibilita à pessoa tomar medidas para lidar com a ameaça subjacente à situação de perigo (Kaplan, Sadock \& Grebb, 1997), as consequiências que altos níveis de ansiedade podem acarretar ao desenvolvimento da criança justificam a adoção de medidas terapêuticas e preventivas.

Se atualmente já há uma vasta literatura abordando as reações depressivas e ansiosas das mães de filhos prematuros, ainda são bastante escassos os trabalhos referentes às mães de crianças com malformações, principalmente quando elas são visíveis, e como evoluem esses estados emocionais após a alta hospitalar, quando há um convívio mais intenso entre mãe e filho. Alguns estudos dão conta que, com o passar do tempo, na grande maioria das famílias, vai ocorrendo uma adaptação gradual, com uma diminuição da ansiedade e um aumento de habilidade em cuidar e aproveitar situações prazerosas (Brunhara \& Petean, 1999; Drotar \& cols., 1975).

O presente estudo teve por objetivo comparar os sinais de ansiedade e depressão entre as mães de recém-nascidos com malformações visíveis em dois momentos, logo após o nascimento do bebê e cerca de três meses após a alta hospitalar.

\section{Método}

\section{Participantes}

Inicialmente os participantes eram 24 mães de recémnascidos com malformação física aparente internados na UTI e UCI Neonatal do Hospital das Clínicas da FMB-Unesp. Cinco mães foram excluídas devido ao óbito do filho antes da alta hospitalar e duas mães desistiram de participar do segundo momento da pesquisa, finalizando com a participação de 17 mães. A maioria das mães era jovem, tinham até 22 
anos de idade $(47,1 \%)$, primigestas $(47,1 \%)$ e todas viviam com o pai do bebê. Com relação à ocupação, 64,7\% das mães eram donas de casa e, as que trabalhavam fora tinham ocupações consideradas mão-de-obra não qualificada ou de qualificação inferior. Quanto à escolaridade, a maioria das mães havia completado o ensino fundamental $(52,9 \%)$. A religião predominante era católica $(70,6 \%)$.

Com relação aos recém-nascidos, o tempo de internação variou de três a 120 dias, com uma mediana de 23 dias. A maioria tinha malformações no sistema nervoso central, como mielomeningocele e hidrocefalia $(41,2 \%)$, ou defeitos do sistema gastrintestinal: gastrosquise e onfalocele $(29,4 \%)$. Os defeitos da face (hemangioma e higroma) acometiam 23,5\% das mães, e havia um caso de acondroplasia.

\section{Instrumentos e medidas}

Os instrumentos utilizados para avaliar o estado emocional materno foram:

1- Inventário de Depressão de Beck (BDI), com tradução e adaptação brasileira de Cunha (2001). O BDI é uma escala de auto-avaliação de manifestações comportamentais de depressão, porém sem finalidade diagnóstica. Esse instrumento é composto por 21 categorias de sintomas e atitudes, com quatro ou cinco afirmações, cada uma refletindo um grau crescente de gravidade de depressão (de 0 a 3) (Gorenstein \& Andrade, 2000). A versão em português do BDI resultou de uma formulação consensual da tradução do original em inglês, que contou com a colaboração de quatro psicólogos clínicos, quatro psiquiatras e uma tradutora, sendo testada junto com a versão em inglês em 32 pessoas bilíngües, com três dias de intervalo e variando a ordem da apresentação dos dois idiomas nas duas metades da amostra. A consistência interna do BDI foi de 0,84 e a correlação entre teste e reteste foi de 0,95 ( $p<0,001)$ (Cunha, 2001).

2 - Inventário de Ansiedade Traço-Estado (IDATE) é um instrumento que pode ser utilizado com adultos normais, como medida objetiva de estado (A-estado) e traço de ansiedade (A-traço), composto por 20 itens que o indivíduo deve responder, avaliando a si mesmo, em uma escala de quatro pontos. As correlações entre a escala original e a brasileira variaram de 0,06 a 0,58 , com uma média de 0,34 para A-estado e de 0,24 a 0,92 com uma média de 0,51 para A-traço. Sua fidedignidade no teste-reteste foi de 0,74 para A-estado e 0,83 para A-traço. A consistência interna Alfa A-estado masculino, obteve o valor de 0,93 e feminino de 0,88 , enquanto que o A-traço masculino obteve o valor de 0,93 e feminino de 0,87 (Spielberger, Biaggio \& Natalício, 1979).

3- Prontuários médicos da mãe e da criança

\section{Procedimento}

O presente estudo foi aprovado pelo Comitê de ética em Pesquisa da Faculdade de Medicina de Botucatu, da Universidade Estadual Paulista. Todas as participantes assinaram o Termo de Consentimento Livre e Esclarecido.

Após o diagnóstico, notificado para a mãe pelo pediatra, verificava-se se a própria mãe notava a malformação. Em caso afirmativo, convidava-se a mãe a participar da pesquisa.
No primeiro contato, eram explicados os objetivos do estudo e, após o consentimento das mães, realizava-se uma entrevista informal e eram aplicados os testes. A fim de neutralizar a interferência da aplicação de um teste sobre o outro, foi realizado um balanceamento, alterando a ordem de aplicação de cada instrumento de avaliação. Dessa forma, para metade dos sujeitos de cada grupo, foi aplicado inicialmente o BDI, depois o IDATE. A outra metade respondeu primeiro ao IDATE, depois ao BDI.

A coleta de dados ocorreu no hospital, enquanto o bebê encontrava-se internado na UTI e UCI Neonatal, de três a sete dias após o nascimento. No segundo momento da pesquisa, de um a três meses após a alta hospitalar, na primeira consulta agendada com a mãe, ela era novamente abordada em seu retorno ao ambulatório, no mesmo hospital.

Paralelamente à realização do processo de avaliação das mães, os dados foram complementados com consultas aos prontuários médicos da criança e da mãe para a obtenção de algumas informações sobre a criança: sua condição de nascimento, a evolução durante o período de sua internação e suas condições de saúde. No prontuário materno, verificou-se o histórico gestacional, parto e condições de saúde pregressa.

\section{Análise dos dados}

Os instrumentos de avaliação dos níveis de ansiedade e depressão foram corrigidos conforme as respectivas normas dos testes. A seguir, foram analisadas as medidas de tendência central (medianas) dos escores obtidos pelas mães nas duas sub-escalas do IDATE e no BDI. Para o cálculo das medianas dos escores maternos, foram utilizados os escores normalizados no IDATE e os escores brutos no BDI, conforme as normas dos testes. Para realizar a análise comparativa dos estados emocionais maternos nos dois momentos, durante o período de hospitalização e após a alta hospitalar do bebê, utilizou-se o Teste não paramétrico de Wilcoxon para amostras pareadas (comparação intra-grupo).

Verificou-se a distribuição das mães de acordo com escores indicativos de sintomas clínicos de ansiedade, disforia e depressão, quantificando-se o número de mães que apresentaram escores indicativos de sintomas clínicos. No IDATE, utilizou-se, como indicativo de sintomas de ansiedade em nível clínico, o critério de corte de escore igual ou acima do percentual 75, já utilizado em outros estudos (Padovani \& cols., 2004). No BDI, por sua vez, foram utilizados os critérios sugeridos para pacientes não diagnosticados com transtornos psiquiátricos, ou seja, disforia $>15$ e depressão>20 (Kendall, Donnell \& Ronamk, 1987). Os resultados quanto à avaliação de sinais clínicos maternos, durante a internação do bebê na UTIN/UCI e após sua alta hospitalar, foram comparados por meio do teste de McNemar.

A fim de verificar possíveis influências de variáveis intervenientes nos resultados, foram realizadas correlações entre as pontuações obtidas no IDATE e no BDI e as variáveis maternas (idade, número de filhos, escolaridade e religião) e a variável do bebê (localização corporal da malformação), a partir do Teste Exato de Fisher. 
Tabela 1. Características das mães.

\begin{tabular}{|c|c|c|}
\hline & $f$ & $\%$ \\
\hline \multicolumn{3}{|l|}{ Idade em anos } \\
\hline Até 22 anos & 8 & $(47,1)$ \\
\hline 23-32 anos & 5 & $(29,4)$ \\
\hline $33-42$ anos & 4 & $(23,5)$ \\
\hline \multicolumn{3}{|l|}{ Escolaridade } \\
\hline Ensino Fundamental Incompleto & 6 & $(35,3)$ \\
\hline Ensino Fundamental Completo & 9 & $(52,9)$ \\
\hline Ensino Médio Incompleto & 0 & $(0)$ \\
\hline Ensino Médio Completo & 1 & $(5,9)$ \\
\hline Ensino Superior Incompleto & 1 & $(5,9)$ \\
\hline \multicolumn{3}{|l|}{ Ocupação } \\
\hline Estudante & 0 & $(0)$ \\
\hline Do lar & 11 & $(64,7)$ \\
\hline Não-qualificada & 4 & $(23,5)$ \\
\hline Qualificação Inferior & 2 & $(11,8)$ \\
\hline Qualificação Inferior & 0 & $(0)$ \\
\hline \multicolumn{3}{|l|}{ Paridade } \\
\hline 1 filho & 8 & $(47,1)$ \\
\hline 2 filhos & 4 & $(23,5)$ \\
\hline 3 ou mais filhos & 5 & $(29,4)$ \\
\hline \multicolumn{3}{|l|}{ Religião } \\
\hline Católica & 12 & $(70,6)$ \\
\hline Evangélica & 1 & $(5,9)$ \\
\hline Outras & 0 & $(0)$ \\
\hline \multicolumn{3}{|l|}{ Estado civil } \\
\hline Casada & 5 & $(29,4)$ \\
\hline Amasiada & 12 & $(70,6)$ \\
\hline Solteira & 0 & $(0)$ \\
\hline
\end{tabular}

\section{Resultados}

A comparação das medianas dos escores de ansiedade (estado e traço) e disforia/depressão das mães em dois momentos distintos, durante a hospitalização e após a alta, mostrou que houve uma redução, da primeira para a segunda avaliação, entre as medianas das pontuações nas três subescalas, e que essa redução foi estatisticamente significativa quanto à ansiedade-estado e disforia / depressão (Tabela 2).

Com relação aos escores indicativos de sinais clínicos, apenas houve uma redução significativa do número de mães que pontuaram para sinais clínicos de ansiedade-estado, da primeira para a segunda avaliação (Tabela 3 ).

Nenhuma associação entre as pontuações nas duas escalas (IDATE e BDI) e variáveis maternas tais como idade, número de filhos, religião e a variável do bebê, local da malformação, foi estatisticamente significativa.

\section{Discussão}

Logo após o nascimento, os níveis de ansiedade situacional das mães de crianças com malformações visíveis foram altos e mais da metade das participantes $(52,94 \%)$ apresentou sinais de ansiedade em nível clínico. Esses níveis de ansiedade situacional podem ter sido influenciados por traços de personalidade, já que $23,5 \%$ das mães pontuaram para ansiedade/traço, mas, possivelmente, também estão associados
Tabela 2. Mediana, 1ํ e $3^{\circ}$ quartis (entre colchetes) dos indicadores de ansiedade e depressão antes e cerca de três meses após a alta hospitalar.

\begin{tabular}{|c|c|c|c|}
\hline Variável & 1a Avaliação & 2a Avaliação & Valor de $p$ \\
\hline & $(n=17)$ & $(n=17)$ & \\
\hline $\begin{array}{c}\text { Ansiedade- } \\
\text { estado } \\
\text { (IDATE) }\end{array}$ & $\begin{array}{c}57[49,0 ; \\
63,0]\end{array}$ & $46[38,5 ; 54,0]$ & 0,002 \\
\hline $\begin{array}{c}\text { Ansiedade- } \\
\text { traço (IDATE) }\end{array}$ & $50[41,5 ; 55,5]$ & $43[39,5 ; 53]$ & 0,115 \\
\hline $\begin{array}{c}\text { Disforia/ } \\
\text { Depressão } \\
\text { (BDI) }\end{array}$ & $12[6,0 ; 20,0]$ & $6[3,0 ; 14,0]$ & 0,040 \\
\hline
\end{tabular}

Tabela 3. Frequiência $(f)$ e taxa de incidência $(i)$ de indicadores clínicos de ansiedade-estado, ansiedade-traço e disforia/depressão avaliados por meio do IDATE e do BDI, respectivamente, na $1^{\underline{a}}$ avaliação $(n=17)$ e na $2^{\underline{a}}$ avaliação $(n=17)$.

\begin{tabular}{cccc}
\hline $\begin{array}{c}\text { Sintomas } \\
\text { Clínicos }\end{array}$ & 1 a Avaliação & 2a Avaliação & Valor de p \\
\hline & $(n=17)$ & $(n=17)$ & \\
$f(\%)$ & $f(\%)$ & 0,04 \\
\hline $\begin{array}{c}\text { Ansiedade- } \\
\text { estado } \\
\text { (IDATE) }\end{array}$ & $9(52,94 \%)$ & $3(17,65 \%)]$ & 1,00 \\
$\begin{array}{c}\text { Ansiedade- } \\
\text { traço (IDATE) } \\
\text { Disforia/ } \\
\text { Depressão } \\
\text { (BDI) }\end{array}$ & $4(23,53 \%)$ & $3(17,65 \%)$ & 0,47 \\
\hline
\end{tabular}

Teste de McNemar

às condições de vida da criança e ao seu encaminhamento para a UTI, logo após o parto. Segundo Britton (2005), mães ansiosas no pós-parto apresentaram uma redução na capacidade de enfrentamento, diminuição da reatividade e sensibilidade e sentimentos de ineficácia no desempenho do papel. No caso das crianças encaminhadas à UTI, a percepção de inadequação como cuidadora se agrava, pois a separação dificulta o contato direto da mãe com a criança e os cuidados estão geralmente a cargo da enfermagem (Quentel, 1997).

Os níveis clínicos de depressão dessas mães, logo após o parto, também estavam bem acima da taxa de prevalência em depressão puerperal na população geral (Moraes \& cols., 2006), mas menor que a incidência encontrada em outros estudos com bebês de risco, como, por exemplo, na pesquisa de Davis, Edwards, Mohay e Wollin (2003), em que 40\% das mães de crianças prematuras apresentaram sinais depressivos, em avaliação realizada entre o primeiro e segundo dia após o parto. Além do fato de essas crianças apresentarem quadros graves e permanecerem na UTI, a depressão materna, também, pode estar associada à percepção de problemas futuros que a criança terá de enfrentar, como cuidados especiais, retornos constantes aos serviços de saúde e a necessidade de acesso a recursos comunitários, ainda deficitários nas pequenas cidades de onde provém a grande maioria dos sujeitos da amostra.

No caso de crianças com malformações visíveis, em especial com defeitos que atingem a face, a essas preocupações se somam o medo dos preconceitos e a preocupação com 
sua inserção social. Desde a Antigüidade, há preconceitos que relacionam traços de caráter e tipos de personalidade às malformações congênitas (Sayler \& Kolb, 1995). Apesar dos recentes avanços em cirurgias crânio-faciais e dos programas de estimulação, que têm aumentado, em muito, as possibilidades dessas crianças atingirem o máximo do seu potencial intelectual e facilitado sua aceitação social, persiste, nos dias de hoje, uma expectativa de que crianças com alterações faciais tenham inteligência subnormal (Campis, 1991).

A condição sócio-econômica das mães é outro fator que pode explicar os níveis de depressão. Estudos recentes, com amostras brasileiras de população de baixa renda, encontraram taxas de prevalência de depressão puerperal acima de 30,0\% (Coutinho \& cols., 2002; Cruz \& cols., 2005). Apesar de esses estudos terem utilizado outros instrumentos de avaliação, o fato de as participantes desta pesquisa serem pacientes de um serviço vinculado ao Sistema Único de Saúde reforça a idéia de que outros eventos vitais que as puérperas das classes menos favorecidas têm de enfrentar logo após o parto podem ser fatores que potencializam o aparecimento de quadros depressivos e merecem maior atenção nos próximos estudos.

Confirmando os estudos com outras crianças de risco, como prematuros (Padovani \& cols., 2004) e crianças com displasia broncopulmonar (Zanardo \& Freato, 2001), houve uma redução estatisticamente significativa das medianas dos escores de ansiedade-estado e depressão das mães na segunda avaliação e de sintomas clínicos de ansiedade-estado, quando a criança não corria mais risco de vida, estava fora do hospital e a mãe tinha mais tempo de convívio e de familiaridade com ela. A diminuição dos níveis de ansiedade e depressão também pode estar associada ao fato de que todas as mães contavam potencialmente com um companheiro. Cruz e cols. (2005) observaram que a presença de suporte por parte do marido apresentou um efeito protetor para depressão puerperal em mulheres atendidas pelo Programa de Saúde da Família. Se, por um lado, a hospitalização do bebê constitui uma situação imprevisível para a mãe, que a expõe à falta de controle dos acontecimentos e, portanto, é altamente ansiogênica (Kennel \& Klaus, 1993), a presença de suporte social pode constituir-se em fator de proteção e facilitar o enfrentamento (Sloper \& Turner, 1993). Segundo Antoniazzi, DellÁglio e Bandeira (1998), a disponibilidade de recursos a que a pessoa sob estresse pode lançar mão, sejam eles pessoais (saúde física, moral, crenças, experiências prévias) ou sócio-ecológicos (redes sociais, circunstâncias econômicas e características familiares), afeta a avaliação do evento estressante e mobiliza um conjunto de esforços cognitivos e comportamentais para adaptar-se à situação adversa.

Outra hipótese que pode explicar a redução significativa dos níveis de ansiedade e de depressão se refere ao fato de que, durante a internação da criança, as mães puderam participar de um grupo semanal que tem por objetivo oferecer suporte psicológico. A constatação dos altos índices de perturbações emocionais nas mães cujos filhos nascem com problemas, especialmente se têm de permanecer em UTIS, levou os hospitais à criação de serviços que promovem o suporte psicológico aos pais de bebês internados, para neutralizar os efeitos adversos dos estressores e atender às necessidades das mães (Doering, Moser \& Dracup, 2000;
Melnick \& cols., 2001). Linhares e cols. (2000), estudando mães de crianças prematuras, afirmam que, em atividade de grupo, essas mães podem conversar sobre suas preocupações, compartilhando da experiência com outras mães, que se encontram em estágios diferentes com relação à evolução de seus bebês, recebendo orientações que ajudam no enfrentamento satisfatório nesse período de crise.

Apesar da redução de mães com sintomas clínicos de depressão, na segunda avaliação, ela não foi estatisticamente significativa. A persistência de sinais clínicos de depressão e necessidade de atendimento psicológico em três mães, quando a criança já dava sinais de melhora clínica e posterior à alta, possivelmente está associada às incertezas geradas pelos cuidados que requerem crianças com problemas de desenvolvimento ou que sofreram intervenções cirúrgicas recentes. A alta incidência de quadros que afetam o sistema nervoso central $(41,2 \%)$ e mesmo a ocorrência de várias cirurgias, nos casos de gastrosquise e onfalocele $(29,4 \%)$, possivelmente, acarretaram atrasos no desenvolvimento, especialmente motor, que, segundo Sloper e Turner (1993), são facilmente identificados pelos pais e prejudicam, nos primeiros meses de vida, a adaptação ao filho portador de problemas.

Algumas limitações metodológicas deste estudo merecem considerações. Em primeiro lugar, o reduzido número de participantes. Tentou-se contornar esse problema desenvolvendo o trabalho em um hospital-escola de nível terciário, que conta com uma UTI de berçário para onde são encaminhados os recém-nascidos de alto risco da região. Conta, também, com um Serviço de Medicina Fetal de referência que recebe as grávidas portadoras de fetos com malformação, que, em geral, acabam dando a luz nesse hospital. De 2002 a 2004, o serviço teve uma procura crescente de crianças nascidas com malformações diversas, com uma média anual de 18 crianças internadas com malformações visíveis, nos três anos anteriores à pesquisa. Em se tratando de estudo longitudinal, os óbitos ocorridos durante a permanência na UTI e após a alta inviabilizaram dados já coletados na primeira entrevista. Assim, o número final de participantes foi pequeno, bastante heterogêneo, seja com relação à visibilidade das malformações, seja quanto à gravidade dos quadros (tomando como parâmetro o tempo de internação das crianças) e acabou não permitindo análises estatísticas mais consistentes de variáveis que podem ter forte associação com os níveis de ansiedade e depressão materna.

Não foi possível também manter o mesmo intervalo de tempo entre a alta hospitalar e a segunda entrevista, para todas as participantes. Estava previsto que a segunda entrevista ocorreria um mês após a alta, quando do primeiro retorno da criança do hospital, tempo que se julgou suficiente para que a mãe tivesse uma percepção da melhora física do filho e tivesse exercido seu papel de cuidadora. Devido à falta das mães à consulta ou remarcações do hospital, algumas mães realizaram a segunda avaliação até três meses após a alta. Possivelmente, esse fato pode ter afetado os resultados tanto de forma positiva como negativa. As mães que foram avaliadas após um período mais longo da alta hospitalar podem ter apresentado níveis mais baixos de ansiedade e depressão, por ter fortalecido o vínculo com o filho e testado sua competência nos cuidados. Por outro lado, esses níveis podem ter aumentado, com a constatação dos atrasos de desenvolvimento da criança e as condições especiais de que ela 
necessita (Sloper \& Turner, 1993). Parece uma variável que precisa ser melhor controlada em próximas pesquisas.

\section{Considerações Finais}

Os dados deste estudo parecem mostrar que o nascimento de uma criança com malformações visíveis provocou, em um número elevado de mães, sinais clínicos de ansiedade e depressão, o que parece reforçar a idéia da importância de suporte psicológico a essas mães quando da internação dos filhos. Confirmando a literatura, com estudos de mãe de crianças de risco, houve uma diminuição significativa dos níveis de ansiedade e depressão após a alta do bebê. Possivelmente está ocorrendo uma adaptação gradual dessas mães, que contam com suporte social, à medida que têm um contato mais íntimo com a criança, percebem suas habilidades como cuidadora (Fagan \& Schor, 1993; Sloper \& Turner, 1993). Investigações futuras deveriam ser orientadas para aprofundar a identificação dos fatores que potencializam ou dificultam a adaptação materna. Tais informações podem ser úteis aos serviços que atendem puérperas de risco, objetivando diminuir ou prevenir a desadaptação ao filho.

A adaptação inicial, verificada nos três primeiros meses após a alta, não significa, entretanto, que essas mães não apresentarão outros episódios de ansiedade e depressão no curso do desenvolvimento da criança. Para Sloper e Turner (1993), o conceito de adaptação a uma criança com deficiências é visto, hoje, como um processo que acompanha toda a vida da criança e não se resume a uma aceitação do diagnóstico, nos primeiros meses de vida. São particularmente estressantes as ocasiões em que os pais percebem a discrepância entre os marcos normais de desenvolvimento e o desempenho de seus filhos, assim como a dificuldade de utilizar os recursos institucionais disponíveis, o que muitas vezes, já ocorre no fim do primeiro ano de vida. Essas observações apontam para a necessidade de estudos longitudinais com um tempo maior de seguimento. Com relação à intervenção, sugere-se que, ao lado de serviços de reabilitação e educacionais especializados, esses pais possam contar com locais para obter informações de recursos comunitários, seja de clínicas ou escolas, assim como a oportunidade de poder participar de grupos de discussão e compartilhamento de experiências, com acompanhamento de profissionais com o objetivo de assegurar o maior aproveitamento das potencialidades de seu filho (Sloper \& Turner, 1993).

\section{Referências}

Antoniazzi, A. S., Dell' Aglio, D. D. \& Bandeira, D. R. (1998). O conceito de coping: uma revisão teórica. Estudos de Psicologia, 3(2), 273-294.

Barnett, B., Schaafsma, M., Guzman, A. \& Parker, G. (1991). Maternal anxiety: a 5- year review of an intervention study. Journal of Child Psychology and Psychiatry; 32, 423-438.

Britton, J. R. (2005). Pre-discharge anxiety among mothers of well newborns: Prevalence and correlates. Acta Pediatrica, 94, 1771-1776.

Brunhara, F. \& Petean, E. B. L. (1999). Mães e filhos especiais: reações, sentimentos e explicações à deficiência da criança. Paidéia, 9(16), 31-40.
Campis, L. B. (1991). Children with Apert Syndrome: developmental and psychological considerations. Clinics in Plastic Surgery, 18(2), 409-416.

Chatterton, R. T., Hill, P. D., Alday, J. C., Hodjes, K. R., Belknap, S. M. \& Zinaman, M. J. (2000). Relation of plasma oxytocin and prolactin concentrations to milk production in mothers of preterm infants: influence of stress. Journal of Clinical Endrocrinology \& Metabolism, 85(10), 3661-3668.

Coutinho, D. S., Baptista, M. N. \& Morais, P. R. (2002). Depressão pósparto: prevalência e correlação com o suporte social. Infanto - Revista Neuropsiquiatra da Infância e Adolescência, 10(2), 63-71.

Cruz, E. B. S., Simões, G. L. \& Faisal-Cury, A. (2005). Rastreamento da depressão pós-parto em mulheres atendidas pelo Programa de Saúde da Família. Revista Brasileira de Ginecologia e Obstetrícia, 27(4), 181-188.

Cunha, J. A. (2001). Manual da versão em português das Escalas Beck. São Paulo: Casa do Psicólogo.

Davis, L., Edwards, H., Mohay, H. \& Wollin, J. (2003). The impact of very premature birth on the psychological health of mothers. Early Human Development, Amsterdam, 73, 61-70.

Doering, L. V., Moser, D. K. \& Dracup, K. (2000). Correlates of anxiety, hostility, depression, and psychosocial adjustment in parents of NICU infants. Neonatal Network, 19(5), 15-23.

Drotar, D., Baskieuvicz, B. A., Irvin, N., Kennel, J. \& Klaus, M. (1975). The adaptation of parents to the birth of an infant with a congenital malformation: a hypothetical model. Pediatrics, 56(5), 710-717.

Fagan, J. \& Schor, D. (1993). Mothers of children with spine bifida: factors related to maternal psychosocial functioning. American Journal of Orthopsychiatry, 63(1), 146-152.

Faisal-Cury, A. \& Menezes, P. R. (2006). Ansiedade no puerpério: prevalência e fatores de risco. Revista Brasileira de Ginecologia e Obstetrícia, 28(3), 171-178.

Frizzo, G. B. \& Piccinini, C. A. (2005). Interação mãe-bebê em contexto de depressão materna: aspectos teóricos e empíricos. Psicologia em Estudo, 10(1), 47-55.

Gorenstein, C. \& Andrade, L. (2000). Inventário de depressão de Beck- Propriedades psicométricas da versão em português. Em C. Gorenstein, L. H. S. G. Andrade, A.W. \& Zuardi (Orgs.), Escalas de avaliação clínica em Psiquiatria e Psicofarmacologia (pp. 89-95). São Paulo: Lemos-Editorial.

Julian-Renier, C., Aurran Y., Dumaret A., Maron A., Chabal F., Giraud F. \& Aymé S. (1995). Attitudes toward Down's síndrome: follow up of a cohort of 280 cases. Journal of Medical Genetics, 32, 597-599.

Kaplan, H. I., Sadock, B. J. \& Grebb, J. A. (1997). Compêndio de psiquiatria: ciência do comportamento e psiquiatria clínica. (7 ed., D. Batista, Trad.). Porto Alegre: Artes Médicas.

Kasak, A. E., \& Clark, M. W. (1986). Stress in families of children with mylomeningocele. Developmental Medicine and Child Neurology, 28, 220-227.

Kendall, P. C., Smith, T. W., Donnell, C. \& Ronamk, K. (1987). Cognitive specificity in emotional distress. Journal of Personality and Social Psychology, 53(4), 734-742.

Kennel, J. H. \& Klaus, M.H . (1993). Atendimento para os pais de bebês prematuros ou doentes. (D. Batista, Trad.). Porto Alegre: Artes Médicas.

Linhares, M. B. M., Carvalho, A. E. V., Bordin, M. B. M., Chimello, J. T., Martinez, F. E. \& Jorge, S. M. (2000). Prematuridade e muito baixo peso ao nascer como fator de risco ao desenvolvimento psicológico da criança. Paidéia, 10(18), 60-69. 
Melnyk, B. M., Alpert-Gills. L., Feinstein, N. F., Fairbanks, E., Schultz-Czarniak, J., Hust, D., Sherman, L., Le Moine, C., Moldenhauer, Z., Small, L., Bender, N. \& Sinkin, R. A. (2001). Improving cognitive development of low-birth-weight premature infants with the COPE program: a pilot study of the benefit of early NICU intervention with mothers. Research in Nursing \& Health, 24, 373-389.

Maldonado, M. T. (2000). Psicologia da gravidez. São Paulo: Saraiva. Moraes, I. G. S., Pinheiro, R. T., Silva, R. A., Horta, B. L., Sousa, P. L. R. \& Faria, A. D. (2006). Prevalência da depressão pós-parto e fatores associados. Revista de Saúde Pública, 40(1), 65-70.

Murray L., Stanley C., Hooper R., King F. \& Fiori-Cowley A. (1996). The role of infant factors in postnatal depression and mother-infant interactions. Developmental Medicine and Child Neurology, 38, 109-119.

Neeldman, R., Walders, N., Kelly, S., Higgins, J., Sofranko, K. \& Drotar, D. (2000). Impacto da triagem para depressão materna em uma clínica pediátrica: um estudo exploratório (M. M. G. B. Mataloun, Trad.). Correios da SBP, 6, 18-19.

Padovani, F. H. P., Linhares, M. B. M., Carvalho, A. E. V., Duarte, G. \& Martinez, F. E. (2004). Avaliação de sintomas de ansiedade e depressão em mães de neonatos pré-termo durante e após hospitalização em UTI-neonatal. Revista Brasileira de Psiquiatria, 26(4), 251-254.

Patel,V., De Souza, N. \& Rodrigues, M. (2003). Postnatal depression and infant growth and development in low income countries: a cohort study from Goa, India. Archives of Disease in Childhood, $88,34-37$.
Pedremônico, M. R. M. (1998). As relações que se estabelecem no triângulo: recém-nascido-família-hospital. Em B. Kolpeman, S. Miyoshi \& S. Guinsburg, S. (Orgs.), Distúrbios respiratórios no período neonatal (pp. 561-563). São Paulo: Ateneu.

Quentel, J.C. (1997) Le probleme de la culpabilité chez les parents d'enfants handicapés. Neuropsychiatrie de L'enfance et de L'adolescence, 45(9), 487-495.

Sailer, H. \& Kolb, E. (1995). Influence of craniofacial surgery on their handling in different cultures and at different times: a contribution to social world history. The Journal of Craniofacial Surgery, 6(4), 314-326.

Schwengber, D. D. S. \& Piccinini, C. A. (2005). A experiência da maternidade no contexto da depressão materna no final do primeiro ano de vida do bebê. Estudos de Psicologia, 22(2), 143-156.

Sloper, P. \& Turner, S. (1993). Risk and resistance factors in the adaptation of parents of children with several physical disability. Journal of Child Psychology and Psychiatry, 34(2), 167-188.

Spielberger, C. D., Biaggio, A. \& Natalício, L. F. (1979). Inventário de ansiedade traço estado: manual de psicologia aplicada. Rio de Janeiro: Cepa.

Zanardo, V. \& Freato, F. (2001). Home oxygen therapy in infants with bronchopulmonary dysplasia: Assessment of parental anxiety. Early Human Development, 65, 39-46.

Recebido em 27.06.2006

Primeira decisão editorial em 28.08.2006

Versão final em 11.10.2007

Aceito em 11.12.2007 